\title{
¿SON LOS VALORES DEL DEPORTE ACTUAL UN MEDIO EDUCATIVO O DE REINSERCIÓN?
}

ARE THE CURRENT SPORT VALUES AN EDUCATIVE AND/OR AN INTEGRATION MEANS?

Manuel Isorna Folgar*

Marta Felpeto Lamas

\section{Resumen}

El deporte y la práctica físico-deportiva son reconocidos como herramientas eficaces y eficientes en las áreas de la educación (tanto formal como informal), la integración de diferentes grupos de población, la rehabilitación y la promoción de la salud. A pesar de la evidencia sobre los efectos positivos alcanzados en varios grupos y áreas de intervención, no se puede negar la naturaleza ambivalente del deporte y que este puede provocar también efectos negativos y perjudiciales. La mercantilización del deporte ha alcanzado límites insospechados hace décadas y ha salpicado a la sociedad en general pero de forma más específica a nuestros niños y jóvenes que tienen a los deportistas como ídolos y referencias personales. En este artículo se hace una crítica a esta situación actual y se plantean una serie de propuestas para reafirmar los valores propios del deporte en edad escolar.

Palabras clave: deporte, mercantilización, valores, práctica deportiva.

\section{Abstract}

Sport and physical and sport practice are recognized as efficient tools in the areas of education (both formal and informal), the integration of different population groups, rehabilitation and health promotion. Despite the evidence on the positive effects in various groups and areas of intervention, there is no denying the ambivalent nature of sports that can also lead to negative and harmful. The commercialization of sport has reached unsuspected limits decades ago and has splashed society in general but more specifically to our children and youth with athletes as idols and personal references. In this article a critique of this situation and will make a number of proposals to reinforce the values of the sport in school.

Keywords: sport, commercialization, values, sports.

Fecha de recepción: 16 de mayo de 2013

Fecha de aprobación: 1 de agosto de 2013

\footnotetext{
* Doctor en Psicología, máster en Drogodependencias, licenciado en Psicología. Docente de la Universidad de Vigo (España). Facultad de Ciencias Educación. Correo electrónico: isorna.catoira@uvigo.es

** Diplomada en Educación Social, Universidad Santiago de Compostela. Correo electrónico: mfelpeto@gmail.com
} 


\section{Introducción}

Un amplio número de investigaciones han confirmado los indudables beneficios físicos (Márquez, RodríguezOrdax y De Abajo, 2006) y psicológicos (Netz, MengJia, Becker y Tenenbaum, 2005) que aporta la actividad física y deportiva practicada de forma regular, produciendo un impacto importante en el bienestar y en la calidad de vida de quienes la practican, independientemente de la edad. También contribuyen al desarrollo de hábitos de disciplina, socialización, conductuales adaptativos, higiénicos y educativos (Ramírez, Vinaccia y Ramón Suárez, 2004), responsabilidad y autocontrol (Cecchini, Montero y Peña, 2003), además de la mejora de la comunicación y la capacidad de relación social (Rossi, Becker y Lancho, 2007), ayuda a reconocer el propio cuerpo y cuidarlo, facilita la resolución de problemas y da sentido de pertenecía a un equipo, generando responsabilidad (Biondi, 2007; Gutiérrez, 2004). Actualmente, al deporte también se le reconoce su capacidad como elemento integrador de colectivos inmigrantes (Heinemann, 2002), medio para enseñar responsabilidad a jóvenes en riesgo (Hellison, 1995), herramienta para la prevención y tratamiento de las drogodependencias (Newcomb y Bentler, 1986), mecanismo favorecedor de la reinserción en las instituciones penitenciarias (Negro, 1995), útil en la recuperación social de los barrios marginales (Balibrea, Santos y Lerma, 2002), favorecedor de la socialización de personas mayores, y activador de diversas funciones en las personas con discapacidad (Goldberg, 1995) y por supuesto como factor protector en la infancia/adolescencia para un mejor desarrollo y ajuste biopsicosocial (figura 1).

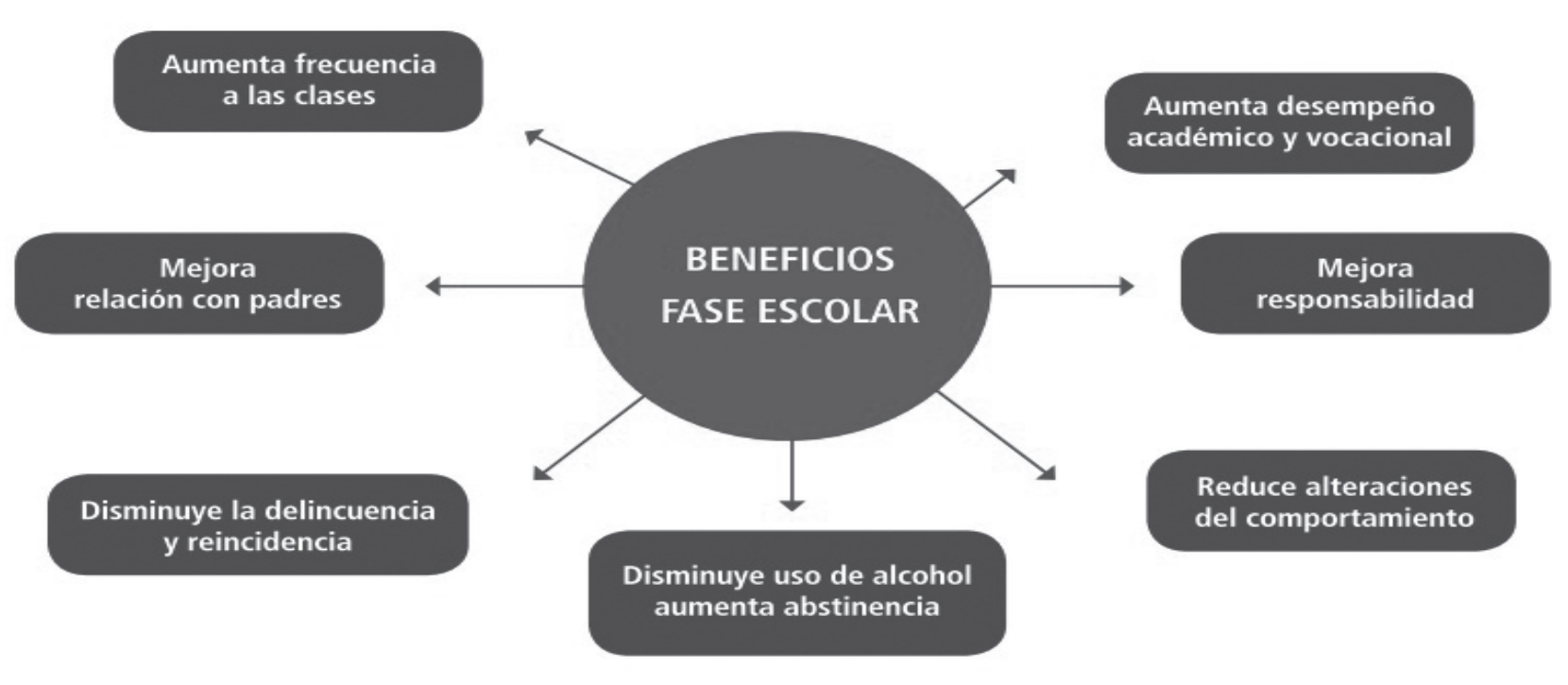

Figura 1. Beneficios de la actividad física en la fase escolar para niños y adolescentes (Matsudo, 2012)

Analizadas las ventajas de la práctica de la actividad física y el deporte no es de extrañar que haya sido empleada en la intervención con poblaciones de riesgo, basando dicha intervención en la promoción de valores sociales y personales (Jiménez, 2006), siendo constatado por la Dirección General de Instituciones Penitenciarias (2005) como el máximo agente de reinserción social en España, que describe al deporte como una de las actividades a llevar a cabo para la rehabilitación de muchas personas. Así mismo, la actividad física fortalece al paciente en su proceso de rehabili- tación por la relación de afectividad que se establece entre el profesional y los pacientes (Biondi, 2007), siendo reconocido como un instrumento muy válido por sus consecuencias terapéuticas para menores en riesgo (Fariña, Vázquez, Mohamed, y Novo, 2009). Sin embargo, puede que no haga falta una investigación tan a fondo para poner en tela de juicio del papel educativo del deporte y nos alcance con echar un vistazo a la prensa deportiva o contemplar uno de los numerosos espacios deportivos de la televisión o radio, o más lamentable todavía, observar el comportamiento de 
muchos niños en el patio del colegio en un recreo para darnos cuenta del tipo de mensaje que se les está transmitiendo e inculcando en estos tiempos tan modernos.

\section{El deporte en la actualidad}

El deporte ha dejado de ser una leyenda social para convertirse en un bien cultural que sirve de emblema a sociedades, regiones y países, punto de unión entre civilizaciones (léase Juegos Olímpicos) y un bien enriquecedor de una sociedad que pretende ser más sana, muchas veces de una forma exacerbada y con una mejor calidad de vida.

El deporte ha pasado a ocupar un primer plano en nuestros días, con una alta consideración social tanto educativa y formativa pero sobre todo a nivel de entretenimiento/espectáculo; esto ha facilitado dos vertientes cada vez más distanciadas en el terreno de sus manifestaciones: por un lado, el deporte espectáculo con la persecución constante de la excelencia, el éxito y el triunfo, y por otro lado, la práctica física y deportiva como medios de educación integral, contacto con la naturaleza, promoción de la salud, alivio del estrés, búsqueda de nuevas sensaciones y aventuras, disfrute y relación social, aspectos lúdicos y formativos más próximos al deporte para todos (Oja y Telama, 1991; Gutiérrez, 2004). Aunque supuestamente estos dos posicionamientos están diferenciados, la realidad es que viene produciéndose una constante invasión de los modelos profesionalizados del deporte adulto, del deporte espectáculo, sobre esos otros modelos más educativos del deporte, aquellos encaminados a convertir el deporte en un estilo de vida activo, saludable y perdurable en el tiempo (Gutiérrez, 2000 y 2004). Esto ocurre, según Knop (1993), porque el deporte destinado a niños y jóvenes se ha visto sometido a una fuerte presión que busca el triunfo por encima de todo, donde ganar es lo único que importa, siendo acompañado por un incremento de la violencia y el engaño.

Por eso, después de numerosos análisis e investigaciones, psicólogos, sociólogos y educadores parecen estar cada vez más de acuerdo en que el deporte será bueno o malo según cómo se desarrolle su práctica. Una gran parte de lo que percibimos y perciben nuestros hijos, niños/jóvenes es un panorama del deporte mercantilizado donde lo único (o casi lo único) que importa es que consuman horas de TV con su respectiva publicidad. Quizá explique ello que hoy en España un tercio de la juventud dedique parte de su tiempo libre a ver deporte por TV (el 32\%, de los cuales ocho de cada diez son chicos) y, en particular, que algo menos de la mitad de los jóvenes vean todas o bastantes retransmisiones deportivas (un $42 \%$, de los cuales seis de cada diez son chicos), según datos de la última encuesta del CIS sobre Hábitos Deportivos en España (2010). De tal modo que la relación entre televisión y deporte queda lejos de cualquier duda; es más, se ha convertido en un binomio dependiente (Moscoso et al., 2010).

El deporte puede enseñar resistencia y estimular un sentimiento de juego limpio y respeto por las normas, un esfuerzo coordinado y la subordinación de los intereses personales a los de grupo; sin embargo, mal utilizado, puede promover la vanidad personal, el deseo codicioso de victoria y odio entre rivales, y un espíritu corporativo de intolerancia y desdén por los demás Así pues, frente a la defensa del valor del deporte como herramienta importante para la educación integral del ser humano, también se levantan voces críticas que resaltan su lado oscuro, el que hace que sus practicantes se vean arrastrados por los modelos del deporte espectáculo y copien lo peor de sus manifestaciones: agresividad, violencia, afán desmesurado de triunfo, frustración ante la derrota y otras cualidades socialmente no deseables (Arnold, 1991; Gutiérrez, 2004).

Ante esta división de opiniones, a lo largo de este artículo presentaremos las cualidades positivas del deporte, a la vez que pondremos de relieve las condiciones en que debe desarrollarse su práctica para que tales efectos positivos puedan alcanzarse. Consideramos que debe servir, entre otras funciones importantes, para reconocerle al deporte su capacidad de múltiples aportaciones a la educación integral del ser humano y como medio para la educación psicoemocional del menor, para resaltar los valores más positivos derivados de su práctica y proponer la eliminación de los aspectos negativos que últimamente se le vienen asociando, dándole un nuevo enfoque en favor de recuperar la verdadera esencia del juego.

\section{Efectos positivos de la práctica físico-deportiva}

Oja y Telama (1991), tras recoger conclusiones de los trabajos presentados en el Congreso Mundial de Deporte para Todos, celebrado en Finlandia en 1990, hacen especial hincapié en el valor del deporte para la formación integral de la persona, es decir, para todas y cada una de sus áreas del desarrollo, tanto para las físicas (forma física, salud y prevención de la enfermedad) como para las psicológicas (autoestima, personalidad, calidad de vida) y las sociales (relación con los demás, 
rendimiento en el empleo, evitación de la soledad), además de poder influir sobre el desarrollo moral y la promoción de diversos valores sociales y personales.

La actividad física nos es inmensamente útil para combatir las enfermedades de moda con alto nivel de mortalidad, como son las cardiovasculares, en cuya etiología se encuentra el sedentarismo y el desequilibrio, tensión física-inacción como factores de alta responsabilidad (García Ferrando, 2001; Bray y Kwan, 2006). Como señala Fox (2000).

la actividad física y el deporte son terapias no farmacológicas efectivas para reducir el estrés, los trastornos del sueño, depresión, ansiedad y otros deterioros que surgen a lo largo del proceso de envejecimiento; al mismo tiempo que aumentan la autoestima y el autoconcepto.

También Biddle (1993) expone que actualmente se reconoce que ciertas formas de actividad física pueden estar relacionadas con el bienestar psicológico y reducir el riesgo de algunos problemas de salud, tanto físicos como mentales, y que la literatura especializada que contempla la investigación sobre los posibles beneficios de salud mental para las personas mayores que practican ejercicio físico con regularidad es cada vez más abundante.

Así, por ejemplo, existe un importante consenso en las afirmaciones hechas por el Instituto Nacional de Salud Mental de Estados Unidos en cuanto a que el ejercicio: se encuentra asociado a la reducción de estados de ansiedad; a largo plazo, reduce manifestaciones y grados de como el neuroticismo; es un buen aliado para el tratamiento profesional de la depresión; favorece la reducción de varios índices de estrés; tiene efectos emocionales beneficiosos a lo largo de todas las edades y para todos los géneros.

Según la información procedente de diferentes publicaciones (Castillo, 1995; Moreno, Cervelló y Moreno, 2008) la investigación desarrollada sobre las posibles repercusiones de la práctica físico-deportiva ha ofrecido los siguientes resultados: la actividad física está relacionada positivamente con la mejora de la autoestima y el autoconcepto además de la salud física, de tal manera que es efectiva para mejorar los aspectos mentales, sociales y físicos de las personas; parece estar relacionada con otras conductas de salud como los hábitos de fumar, la alimentación y la higiene, por lo que aumentando la práctica de actividad física, se podría reducir otros hábitos relacionados con el deterioro de la salud; los años escolares representan un periodo fundamental, sensible y crítico en el desarrollo de hábitos de práctica de actividad física y deportiva.

En esta línea, Ruiz y García-Montes (2002) consideran que el deporte ofrece maravillosos medios para instaurar estilos de vida que sean perdurables, enriquecedores, estimulantes y saludables para toda la vida. Sin duda, el ejercicio físico incrementa el bienestar psicológico a lo largo de la vida, aumenta el autoconcepto y provee de oportunidades para experimentar momentos álgidos. Desafortunadamente, tales beneficios son experimentados por un pequeño porcentaje de gente, aquellos que se ejercitan con cierta frecuencia (Gutiérrez, 2004). También Balaguer y García-Merita (1994) destacaron que la actividad física posee efectos beneficiosos para la salud. En un trabajo desarrollado con muestras españolas, encontraron una relación positiva entre la realización de actividad física de forma regular y la mejora del autoconcepto-autoestima, los estados de ánimo, la mejora de la depresión y de la ansiedad. Además, la actividad física parece estar positiva o negativamente asociada a otras conductas como fumar, dieta e higiene.

La actividad física también parece ejercer efectos positivos sobre otras áreas del desarrollo humano. Field, Diego y Sanders (2001) concluyen que los estudiantes con mayor nivel de ejercicio presentan mejores relaciones con sus padres (en cuanto a intimidad, calidad en las relaciones, frecuencia de manifestaciones afectivas y apoyo familiar), menor depresión, emplean mayor cantidad de tiempo en actividades deportivas, menor uso de drogas y tienen mejor rendimiento académico que los estudiantes inactivos o sedentarios. Como puede apreciarse, son cada vez más numerosos los autores que coinciden en señalar la práctica física regular como un elemento generador de multitud de beneficios psicológicos y sociales. La evidencia acumulada sugiere que para la población general, la actividad física estructurada y planificada está asociada con beneficios psicológicos en cuatro grandes áreas: mejor estado de ánimo, reducción del estrés, autoconcepto más positivo y calidad de vida más elevada. Por supuesto, estos beneficios son aún más destacados en poblaciones especiales como las personas clínicamente depresivas o ansiosas, quienes padecen enfermedades coronarias, los mayores de edad y quienes padecen otras dolencias específicas. 
Además, el ejercicio, si es habitual, también está asociado con una gran cantidad de beneficios físicos. En consecuencia, tanto investigadores como profesionales de la actividad física y la salud buscan formas de aumentar el número de personas que persigan y mantengan estilos de vida activos. Sin embargo, como señalaba Daley (2008), a pesar de la evidencia que apoya la relación entre el ejercicio físico y el bienestar, sobre todo psicológico, la mayoría de las personas permanecen sedentarias. En el pasado, parecía que los jóvenes asumían su condición de sujetos activos, pero el contexto social y los cambios tecnológicos producidos en las últimas décadas han afectado los niveles de actividad física de los niños, hecho que implica que estas actividades sedentarias (televisión, ordenador, videojuegos) y viajar en auto más que en bicicleta. Estos cambios también parecen haber modificado los estilos de vida de los jóvenes y los patrones de actividad física general, hasta el punto de llegar a constituir serios problemas de salud en los países industrializados, sobre todo, con la llegada de la TV y los ordenadores (American Academy of Pediatrics, 2001; Abarca, Casterad, Generelo y Clemente, 2010).

Berger (1996) realiza una reflexión en el sentido de sus observaciones diarias, en cuanto la mayoría de las personas intentan evitar el esfuerzo y la actividad física vigorosa en cualquiera de sus formas. Es difícil comprender cómo las personas a pesar de conocer las bondades del ejercicio físico, haya tan pocas que lo practiquen con suficiente intensidad y frecuencia como para beneficiarse de tales ventajas. En la misma línea, Rainer Martens (1996) sugiere que tal vez el objetivo más importante de los profesionales de la actividad física sea convertir a los niños en practicantes activos para toda la vida. Según este autor, todos queremos que nuestros niños y jóvenes tengan un amplio conocimiento sobre la amplia variedad de actividades físicas, adquieran la habilidad necesaria para implicarse en ellas y sepan apreciar los beneficios de una vida activa. De este modo, el reto de todos los interesados en mejorar la salud pública es conocer cómo impedir la tendencia hacia la inactividad física en los escolares, cómo influir en los niños para que desarrollen hábitos de ejercicio físico y lo trasladen a la vida adulta. De aquí que un objetivo principal de nuestra tarea debería consistir en conocer por qué la mayoría de los adultos son inactivos, y esto solo es posible si partimos de un análisis sobre cómo iniciamos a los niños en la actividad física y el deporte (Gutiérrez, 2000, 2004).

\section{Aspectos negativos de la práctica físico-deporti- va en edad escolar}

\section{Estereotipos y actitudes sexistas}

Cada sociedad va configurando estereotipos asociados al género, la edad, así como a cualquier otra medida como la raza, nacionalidad, etc.; en definitiva, se van interiorizando una serie de formas de sentir, pensar y actuar que determinan papeles sociales diferentes según el sexo (Sicilia, 2002) y sirven como referencia para la atribución de un determinado estatus, la expectativa de rol consecuente con el anterior, así como el interés por practicar un determinado deporte o práctica físico-deportiva. Por consiguiente, la fuerza de estos estereotipos físico-sociales va a repercutir en el hecho de que los alumnos lleguen a la escuela con unas actitudes sexistas, reforzadas por la intervención educativa, llevando todo ello paulatinamente a las adolescentes (figura 2) hacia una desmotivación por la educación física y hacia una serie de consecuencias, como una escasa participación en actividades físico-deportivas extraescolares, una falta de adherencia en programas de estas características durante la etapa poseducativa y un crecimiento de los índices de abandono durante la edad adulta (Moreno, Martínez y Alonso, 2006).

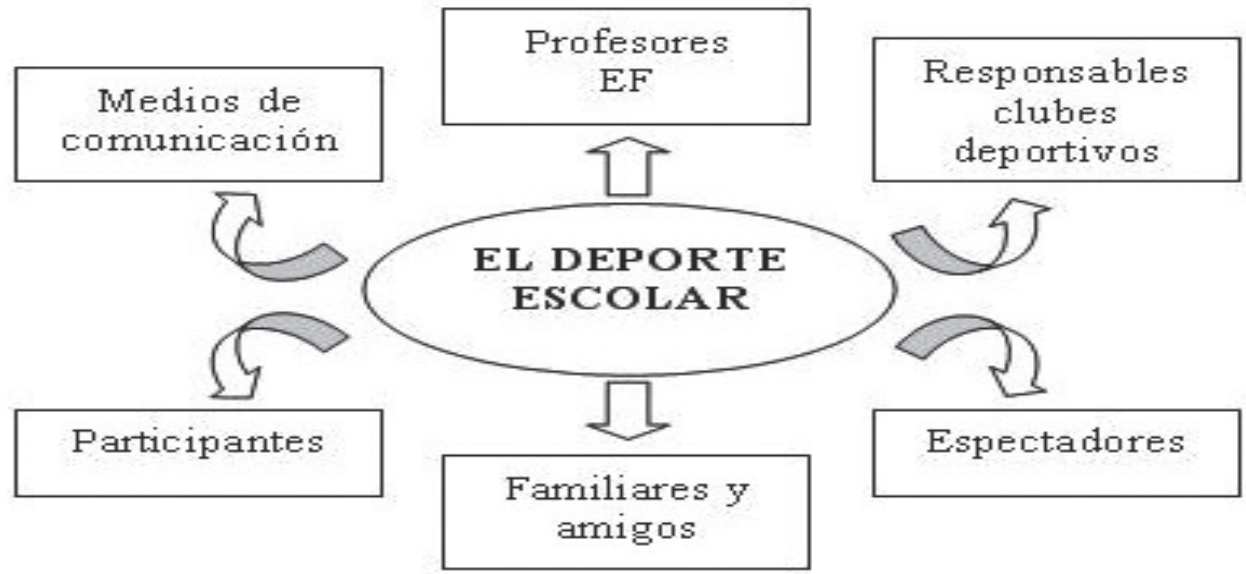

Figura 2. Elementos que influyen en el deporte en edad escolar (Tomás, Robles y Giménez, 2009). 
Algunas de estas actitudes sexistas adquiridas por los alumnos hacen referencia al hecho de que la mayoría de niñas y adolescentes, sobre todo a partir de los 1314 años, rechazan los esfuerzos de cierta intensidad (trabajo de resistencia, potencia, etc.), mientras que los niños, por el contrario, suelen aceptar mejor este tipo de esfuerzos, bien sea por la imitación de determinados deportistas o por la imagen social y familiar implícita en el deporte. Del mismo modo, la mujer adolescente, en general, observa una mayor identificación de la actividad deportiva con el varón que con ella misma, aunque aceptan participar en actividades masculinas en mayor medida que los chicos lo hacen con actividades catalogadas como femeninas, tal es el caso de la expresión corporal o la danza. Otra condición sexista hace mención al hecho de que la propia mujer se sitúe en condiciones de inferioridad en cuanto a sus posibilidades de aprendizaje, lo que determina la falta de motivación femenina hacia la actividad física. Debido a esta falta de motivación, así como por el complejo de inferioridad, la mujer evita entrar en competencia con el sexo opuesto en el campo de la actividad física, un sexo seguro de sus posibilidades y con liderazgo. Otro de los motivos que puede condicionar la interacción entre alumnos en el desarrollo de actividades físicas es el nivel de agresividad física a través de contactos corporales reglados que suelen ser mucho más utilizados por los niños que por las niñas. Por último, la menstruación, los efectos negativos sobre el aparato reproductor femenino y la masculinización de la mujer, han sido los grandes temas sobre los que se ha asentado la inhibición deportiva de la mujer y, aunque en la actualidad dichos prejuicios parecen carecer de sentido, las adolescentes suelen emplearlo como excusa para rechazar la realización de actividad físico-deportiva (Moreno, Martínez y Alonso, 2006).

\section{La competitividad}

La actividad competitiva puede generar entre los escolares comportamientos agresivos relacionados con los eventos deportivos e influenciados por la necesidad de conseguir resultados, la actitud violenta de algunos socios ultras, la información que producen algunos medios de comunicación social y aquellos entrenadores que estimulan y demandan comportamientos agresivos a los deportistas. Es decir, conductas originadas en la alta competición y que se reproducen, cada vez más, en el deporte escolar (Fraile y De Diego, 2006).

Para Bandura (1978), la conducta agresiva es aprendida por medio de la imitación en experiencias de los escolares; considerando que no se nace con un repertorio de conductas agresivas, sino que estas se van adquiriendo a lo largo de su desarrollo. Por tanto, el deporte, como actividad basada en un gran número de comportamientos competitivos y agresivos, puede llegar a ser un referente de gran influencia para los escolares (desde la televisión se repiten imágenes con conductas agresivas de los jugadores, perjudiciales para los escolares).

Según Buceta (1998), la competición puede producir en el deportista una serie de situaciones estresantes, que son transferidas a las etapas escolares. Ante esto, los educadores deben enseñar a actuar a los escolares frente a situaciones como: la trascendencia y la incertidumbre del resultado; la dificultad de predecir el propio rendimiento; la posibilidad de no alcanzar el resultado deseado; las consecuencias negativas que pueden derivarse del fracaso; competir en circunstancias desfavorables; el esfuerzo por encima de sus posibilidades; las decisiones adversas de los árbitros; la conducta exigente del entrenador y del entorno familiar.

En el Código de Ética Deportiva del Consejo Superior de Deportes se dice que todos los niños tienen derecho a competir y a divertirse, pero que la competición debe ser y estar adaptada a las edades de nuestros alumnos. Por su parte, González (1993) apunta que el niño está preocupado por mejorar sus destrezas motrices y por la competición, sugiriendo que en la programación didáctica debemos utilizar el juego competitivo como medio de introducir al niño en la resolución de problemas motrices. Por tanto, la competición en sí no es negativa, sino su mala interpretación y aplicación en la enseñanza. La competición será negativa cuando premiamos los resultados, cuando la hacemos discriminatoria, solo para los mejores, en definitiva, cuando la hacemos trascendente. Sin embargo, sí podemos utilizar la competición como medio educativo cuando no priman los resultados, cuando participan todos, cuando nos sirve como mejora del aprendizaje de nuestros alumnos y cuando no especializamos en edades tempranas en una sola modalidad deportiva.

\section{Actividad física y deporte un proyecto de futuro ineludible}

La Organización Mundial de la Salud (OMS), en su estrategia "Salud para todos en el año 2010", incluye entre sus objetivos la reducción de la prevalencia de sobrepeso y obesidad en todos los grupos de edad, así como el incremento de la proporción de adultos que realiza actividad física moderada diaria, al menos durante 30 minutos; y en adolescentes, promover las actividades físicas que proporcionen una buena capacidad cardio- 
rrespiratoria tres o más veces por semana (USDHHS, 2000). A pesar de todas estas evidencias, la actividad física está poco arraigada en la población, que cada vez se está haciendo más sedentaria. Además, los resultados de los programas emprendidos para fomentarla no han resultado del todo satisfactorios (Elizondo, Guillén y Aguinaga, 2005).

El estilo de vida que predomina en la sociedad española es el sedentario, caracterizado por altos índices de estrés relacionado con carencia de actividad física y que ocasiona diversos problemas psicológicos como ansiedad, depresión, trastornos adictivos, etc., pero sobre todo enfermedades de tipo no infeccioso, es decir, diabetes, enfermedades cardíacas, cáncer, etc. Para contrarrestar estos efectos, numerosos gobiernos han incorporado la actividad física y el deporte en sus programas de promoción de la salud, llevando a cabo acciones para cambiar la actitud y la conducta de sus ciudadanos respecto a la práctica deportiva, de tal manera que la educación físico-deportiva es considerada como un área importante en el desarrollo personal y en la mejora de la calidad de vida de los ciudadanos, como podemos observar en los países nórdicos europeos.

En diferentes foros se viene señalando que la edad escolar representa un periodo crítico en el desarrollo de los hábitos de práctica física los cuales trasladará a la vida adulta. Según diversas estadísticas, la mayoría de los niños de 10 años participan en varios tipos de actividad física, pero esta tasa decrece significativamente a lo largo de la década, de manera que a los 17 años, un $80 \%$ de los jóvenes han abandonado el contexto del deporte. Esto constituye un serio problema ya que la participación en este tipo de actividades por lo general no es reemplazada por ningún otro tipo de ejercicio físico, hecho que no es solo significativo respecto a la salud y bienestar de los niños y adolescentes, sino también en relación con la probable importancia de la actividad física como una parte del estilo de vida saludable del adulto. Iverson et al. (1985) afirmaban que la escuela representa un escenario ideal para influir en la práctica de la actividad física en niños y jóvenes, y que unos buenos programas en esta área en los colegios podrían aportar el conocimiento y las destrezas necesarias para generar estos hábitos a lo largo de la vida.

El desarrollo temprano de prácticas saludables parece ser primordial tanto para la salud infantil como para la calidad de vida de los adultos. Numerosos investigadores han indicado que los patrones de conducta de actividad física se establecen en la vida temprana de los jóve- nes. Es muy importante que los jóvenes se impliquen en programas de práctica física porque la vida sedentaria muy pronto fijará en ellos los esquemas para adoptar una vida también inactiva en su etapa adulta, argumento poderoso por el que deben promocionarse tanto el deporte como la educación física en las escuelas ya que los patrones de ejercicio grabados en la adolescencia permanecen, sin ninguna duda, como el mejor predictor de los niveles de actividad adulta (Sallis y Patrick, 1994). Kelder, Perry, Klepp y Lytle (1994) indicaron que los hábitos adquiridos en edades jóvenes reflejan el tipo de conductas en la vida adulta, que los patrones de conducta consolidados durante la infancia, a menudo, se mantienen durante toda la vida. Por consiguiente, parece lógico que las escuelas deban centrarse en la promoción de la participación de los jóvenes en las prácticas de actividad física y deportiva para la consolidación de estilos de vida activos y perdurables.

Locke (1996) afirma que cualquier estrategia encaminada a promocionar una vida saludable en los adultos a través de la actividad física, no logrará ni siquiera un modesto éxito si no se afronta de lleno lo que ocurre con los niños y adolescentes en la educación física escolar. A menudo. en las clases de educación física, y sin directrices para la preparación de la sensibilidad adulta, los niños se enseñan unos a otros a odiar el deporte y el ejercicio, a devaluarse a sí mismos y a crear estilos de vida sedentarios como formas de escape por encima de la humillación y el miedo.

Las escuelas españolas proporcionan una de las pocas oportunidades de abarcar a todos los individuos, al menos hasta los 16 años dado el carácter obligatorio de la escolarización, para proponerles e inculcarles los programas de actividad física-deportiva, y la última oportunidad de captar a toda la audiencia sin coste adicional (Fox, 1994). Además, la escuela ofrece tres oportunidades principales para que la gente joven sea físicamente activa: juegos durante el recreo; clases de educación física y actividades físico-deportivas extracurriculares.

Una vez que los jóvenes dejan el contexto escolar, estas oportunidades se reducen y las ocupaciones laborales no proporcionan tantas posibilidades de mantenerse físicamente activos. Por consiguiente, parece claro que los profesionales de la actividad física y el deporte necesitan trabajar en favor de la normalización del ejercicio físico y las actividades durante las clases de educación física y actividades extracurriculares, puesto que la actividad física podrá formar parte de los estilos 
de vida de estos jóvenes cuando sean adultos. Nos parece de vital importancia que el centro escolar intente, en la medida de lo posible, establecer relación con los clubes deportivos de localidades cercanas para que los alumnos conozcan y se familiaricen con estas instalaciones, pues una vez concluida la escolarización obligatoria, tendrá un punto de referencia a dónde acudir a realizar actividad física.

Según Martens (1996) hemos aprendido mucho acerca de la fisiología y la psicología de la actividad física en lo que respecta a los niños y jóvenes; hemos reconocido en especial los beneficios saludables, y las habilidades psicológicas y sociales que pueden aprenderse cuando se está implicado en la práctica deportiva y actividades físicas recreativas. También hemos aprendido una considerable cantidad de cosas acerca de los principios del comportamiento humano en general y más específicamente de lo que se refiere a la participación de los jóvenes en actividad física, pero nuestra más seria necesidad es aplicar lo que ahora sabemos.

Como observamos, existe el convencimiento general de que si los niños y adolescentes adquieren estilos de vida activos y saludables, los hábitos instaurados en las primeras edades influirán significativamente marcando la continuidad de este estilo en la edad adulta, y también que si los estilos de vida activos acompañan a los estilos de vida saludables, podremos potenciar éstos a través de la actividad física y el deporte (Gutiérrez, 2000).

Por ello, la exigencia de la planificación de la formación no puede tener una finalidad compensatoria en la que la utilización de la competición se va a realizar siempre como un medio de motivación y aprendizaje de los alumnos y nunca como el fin último a conseguir. Es decir, la importancia de la competición debe recaer en su incidencia en la mejora del proceso de enseñanza/ aprendizaje y nunca en la consecución de resultados a corto plazo. En estas edades vamos a rechazar toda situación en la que la competición sirva como medio de discriminación y selección (Giménez y Castillo, 2001). En este sentido, Martens (1996) alude al poder de las experiencias tempranas de actividad física, a la vez que alerta sobre ciertos peligros, debido a que el deporte juvenil, para algunos al menos, puede ser bastante deseducativo, puesto que los niños no solo pueden aprender lecciones distorsionadas acerca del deporte y el ejercicio, sino que pueden adquirir imágenes de ellos mismos como incapaces y degradantes. Esta es una idea que también ha señalado Daley (1997), quien indica que muchos niños ven el deporte y las activi- dades físicas extraescolares como solo para aquellos que son capaces, talentosos y buenos deportistas, por tanto las rechazan. Tal vez esta imagen se haya desarrollado porque la actividad física en las escuelas se ha basado en la participación en deportes competitivos, algo que aún hoy se mantiene, como puede verse en relación con el deporte en España.

Por eso, Roberts y Brodie (1994) señalaban que el deporte es la actividad de una gran minoría entre una gran mayoría de gente inactiva. Este énfasis en el deporte competitivo puede estar negando a muchas personas la oportunidad de participar en actividades cotidianas de las cuales obtener una satisfacción y la inclinación a continuar activos a lo largo de su vida. Si bien es verdad que estos autores se refieren a contextos ingleses y americanos, no es menos cierto que tales afirmaciones podríamos aplicarlas a muchos de nuestros propios contextos. No debemos olvidar que el significado personal del deporte es muy complejo, de tal manera que una persona puede hacer ejercicio por sentirse mejor, otra puede buscar la excitación y el alto riesgo en el deporte; unos pueden hacer ejercicio para perder peso o mejorar su apariencia y otros para conseguir vivir sanos durante cien años.

\section{Deporte, educación en valores y desarrollo moral}

El valor educativo del deporte es destacado por los psicólogos, pedagogos, sociólogos y educadores físicos como un instrumento importante para el desarrollo psicosocial del individuo y como medio de integración social y cultural. Quienes defienden este planteamiento, consideran el deporte como una herramienta apropiada para enseñar a todos, pero sobre todo a los más jóvenes, virtudes y cualidades positivas como justicia, lealtad, afán de superación, convivencia, respeto, compañerismo, trabajo en equipo, disciplina, responsabilidad, conformidad y otras (Cruz, 2004).

Pero a su vez, no podemos ignorar que cada día son más frecuentes las prácticas deportivas que se olvidan de esos valores y resaltan la vanidad personal, intolerancia, alineaciones ilegales, empleo de drogas para mejorar el rendimiento, conductas agresivas y abundancia de trampas (Shields y Bredemeier, 1995; Gutiérrez, 2003). También señalan algunos autores el excesivo empeño puesto en el triunfo, y que la competición reduce los comportamientos prosociales y promueve conductas antisociales (Bay-Hinitz, Peterson y Quilitch, 1994; Beller y Stoll, 1995; Priest et al., 1999). En este mismo sentido se expresaba Hardman (1998) 
al indicar que en los últimos años se ha producido una clara comercialización del deporte, por lo que algunas escuelas del Reino Unido, ejemplo tradicional del fair-play, han juzgado necesario introducir un código deportivo de conducta para combatir el declive de la deportividad en las competiciones escolares, considerado una consecuencia del deporte de alto nivel.

Pero seamos optimistas y aboguemos por el lado bueno, el que permite obtener cualidades positivas a través de la práctica deportiva, sin olvidar, no obstante, que tanto los propios practicantes como los sistemas organizativos y educativos, a diferentes niveles, deberán mantenerse alerta y poner todos los medios a su alcance para evitar caer en la vertiente no deseable. Son muchos los valores que pueden trabajarse mediante la práctica deportiva. Por ejemplo, valores utilitarios (esfuerzo, dedicación, entrega), valores relacionados con la salud (cuidado del cuerpo, consolidación de hábitos alimentarios o higiénicos), valores morales (cooperación, respeto a las normas). Ahora bien, según Amat y Batalla (2000), para educar en valores, el deporte debe plantearse de forma que permita: a) fomentar el autoconocimiento y mejorar el autoconcepto, c) potenciar el diálogo como mejor forma de resolución de conflictos, c) la participación de todos, d) potenciar la autonomía personal, e) aprovechar el fracaso como elemento educativo, f) promover el respeto y la aceptación de las diferencias individuales, g) aprovechar las situaciones de juego, entrenamiento y competición para trabajar las habilidades sociales encaminadas a favorecer la convivencia.

En consonancia con este planteamiento, podemos señalar que en las dos últimas décadas se han desarrollado diversos programas de intervención destinados al desarrollo de valores en los contextos de la actividad física y el deporte, entre los cuales Shields y Bredemeier (1995) y Weiss y Smith (2002) destacan como más significativos los siguientes: "Fair-play para niños" (Bredemeier et al., 1986; Gibbons, Ebbeck y Weiss, 1995); "Desarrollo de habilidades para la vida" (Danish y Nellen, 1997); "Enseñanza de responsabilidad social y personal" (Hastie y Buchanan, 2000; Hellison, 1995); "Programas de educación sociomoral" (Miller, Bredemeier y Shields, 1997); "Deporte para la paz" (Ennis et al., 1999). En España destaca el programa "El club del buen deportista" (FAD, 2009), el cual pretende desarrollar estrategias preventivas a través de la difusión de la actividad deportiva como fuente de aprendizaje de valores positivos y fórmulas óptimas de ocupación del tiempo libre.
Todos estos programas se basan en dos teorías principales: la del aprendizaje social (Bandura, 1986), y la del desarrollo estructural, fundamentada en el desarrollo del razonamiento moral (Haan, 1978; Kohlberg, 1969). En nuestro propio marco social y educativo, aunque aún resultan insuficientes, cada día son más abundantes los programas de este tipo que están utilizando la educación física y el deporte como recursos para desarrollar valores, la moral y la responsabilidad personal y social (Gutiérrez y Vivó, 2002), obteniendo resultados considerables.

\section{¿Cómo ha de ser la práctica físico-deportiva para que resulte educativa?}

La educación física y el deporte como práctica pedagógica se encuentran estrechamente relacionados con el juego y el recreo; a la vez que ocupan un lugar privilegiado en la vida del niño, ya que le permite conformar y consolidar su proyecto de vida (Jaramillo, 2003). La educación física es un medio integrador y eficaz para transmitir a los niños y jóvenes las habilidades, modelos de pensamiento, conocimientos y valores (Vargas y Orozco, 2004); por ejemplo, tal y como recoge Flores y Zamora (2009) los valores que se encuentran más consolidados en el currículum de educación física en los centros educativos de Granada fueron: cooperación, autonomía, tolerancia y participación; mientras que en Canarias fueron: tolerancia, integración, autonomía y cooperación (Gómez, 2005).

La educación física-deportiva debe superar las concepciones reduccionistas de antaño, y asumirse como una actividad que busca el desarrollo de todas las capacidades de la persona, así como su inclusión en la cultura actual mediante la transmisión y disfrute de los bienes que la constituyen y, sin duda, uno de los elementos que conforman esta cultura es el deporte (Vázquez, 2001). Pero este ha de ser educativo, y para eso, ha de permitir el desarrollo de las aptitudes motrices y psicomotrices en relación con los aspectos afectivos, cognitivos y sociales de la personalidad del individuo (Le Boulch, 1991). En este mismo sentido se expresan Contreras, De la Torre y Velázquez (2001) indicando que el enorme auge que ha tenido en las últimas décadas el deporte centrado en la competición y el rendimiento, su gran difusión a través de los cada vez más omnipresentes medios de comunicación, y su poderosa capacidad de influencia en otras vertientes de la práctica deportiva, constituyen algunos de los factores que han propiciado la transposición acrítica de su significado, formas, métodos y valores al ámbito escolar, lo que ha supues- 
to con frecuencia una desvirtuación del significado y sentido que deben tener tales aspectos en el contexto educativo.

Por eso, tal y como recoge Gutiérrez (2003) al reconocer la trascendencia de la socialización infantil y juvenil mediante los juegos y deportes, el Consejo de Europa comunicó a sus miembros cuáles debían ser, al menos, las funciones que debía cumplir el deporte destinado a los niños y jóvenes:

- Respetar, en su unidad, todos los aspectos de la persona.

- Desarrollar la capacidad de cada cual para evaluar sus propias posibilidades y desarrollar los distintos aspectos de su personalidad en el respeto de sí mismo y de los demás.

- Favorecer una práctica deportiva de ocio en un ambiente de diversión, sin olvidar el rigor del aprendizaje.

- Adoptar una pedagogía del éxito que no conduzca a logros demasiado fáciles o a fracasos de graves consecuencias.

- Proponer un amplio abanico de actividades individuales y colectivas.

- Permitir que cada cual elija las actividades según sus gustos, necesidades y placer que le aporten.

Todo eso es enormemente importante porque algunos de los beneficios asociados con la actividad física incluyen: aprender a depender unos de otros, aumentar la autoestima y autoconfianza, construir un sentimiento de responsabilidad y trabajo en equipo, desarrollar la persona al completo, y adquirir buena deportividad.

Todos los diseños curriculares de casi todos los centros educativos de España recogen en su espíritu la promoción del desarrollo ético, moral, cultural, mental y físico de los alumnos, y que los estudiantes deben dotarse de competencias para aprovechar las oportunidades, posibilidades y experiencias que la vida les reportará en la vida adulta. De aquí que una concepción amplia de la salud (física, psicológica y social) en relación con la educación deportiva permitirá a los profesores promover algunas de estas metas y ayudar a promover el disfrute y la motivación por la actividad física en los niños para que estos se conviertan en adultos activos, lo cual puede suponer una significativa contribución tanto al desarrollo individual de los niños como a la salud pública general (Biddle, 1993).

Devís (1995) proponía una serie de cambios sobre los que debería incidir una verdadera reforma del deporte escolar para que este fuese en realidad educativo: sobre la trascendencia social que se concede a la victoria y el resultado en el deporte; sobre la competición como único valor ligado a la participación deportiva; sobre la rivalidad, competitividad y agresividad que puede surgir en el deporte, y que se oponen a ciertos valores morales como la cooperación, el respeto o la igualdad; sobre la utilización política, ideológica y económica que tiene el deporte.

También reforzando la idea de que el deporte debe cumplir con una serie de requisitos para que resulte educativo, Cruz (2003) expone lo siguiente: "valorar la asistencia a los entrenamientos y competiciones; el esfuerzo; la cooperación con los compañeros; la competición deportiva con los contrarios, respetando el reglamento; el hecho de divertirse y pasárselo bien, por encima de los resultados" (s.p.).

Por tanto, para que el deporte pueda llegar a ser una tarea educativa y de integración social y cultural se deberán revisar los objetivos del deporte en edad escolar, el papel de los padres, técnicos o entrenadores y compañeros, así como árbitros y organizadores de competiciones, sin olvidar los modelos que ofrece el deporte profesional y el tratamiento informativo que de esto hacen los medios de comunicación (Cruz, 2003).

Un aspecto al que se le viene prestando gran atención es el de la agresividad en los contextos deportivos. Los resultados obtenidos en investigaciones, como las de Bredemeier (1995), sugieren que la conducta agresiva en el deporte está relacionada con la atmósfera moral de su equipo, incluyendo normas sobre la agresión, percepción de los jugadores sobre estas normas y las características del entrenador, así como las motivaciones morales de los jugadores para comportarse de una determinada manera. Luego, parece estar en manos de los entrenadores y educadores físicos una gran parte de la respuesta a cómo debe ser el deporte para que resulte educativo. En una investigación desarrollada por Guivernau y Duda (2002), los deportistas manifiestan que están más dispuestos a cometer agresiones si entienden que su entrenador apoya tales conductas.

No debemos olvidar que nuestros jóvenes deportistas pasan muchas horas con sus respectivos entrenadores de club y que esa posible formación deficiente, por parte de las personas que imparten docencia en las actividades físico-deportivas extraescolares, obstaculiza una orientación educativa y de salud de las propias actividades, ya que una adecuada intervención didáctica depende, en esencia, de una apropiada calificación de los profesionales, es decir, de su formación inicial y 
continua (Campos-Izquierdo, 2004; Fraile, 1999). Esto resalta, una vez más, la capacidad de influencia que los otros significativos tienen sobre el clima moral que se crea en los equipos deportivos y las consecuencias que pueden derivarse de sus prácticas.

Otra vertiente que también resulta reveladora para comprender el comportamiento de los deportistas es la que contempla las orientaciones de meta y los climas motivacionales en los contextos de práctica físicadeportiva. En este sentido Tod y Hodge (2001) y Cecchini et al. (2004) exponen que los deportistas cuyo perfil se caracteriza por una mayor orientación al ego, tienden a emplear niveles de razonamiento moral menos maduros, influenciados por los intereses propios y las actitudes de ganar a toda costa. Por el contrario, los deportistas cuyo perfil de metas está formado por una combinación de orientaciones al ego y a la tarea, tienden a emplear mayores niveles de madurez en su razonamiento moral, caracterizándose por una mayor preocupación hacia los demás. Una vez más se comprueba que el razonamiento moral de los deportistas se ve influenciado por variables situacionales tan importantes como el clima creado por los otros significativos. Por eso decía Arnold (1998) que el grado en que las actividades físicas pueden utilizarse de manera instrumental a los efectos de los objetivos de la educación dependerá siempre, en una u otra medida, de la intención, el conocimiento, la imaginación y la destreza del profesor, entrenador o educador físico en general.

En las clases de educación física y en la práctica deportiva en general, se debe tratar de crear un ambiente lo más agradable posible ya que estas experiencias resultarán críticas para el desarrollo de sentimientos tanto positivos como negativos en los niños/adolescentes hacia la actividad física (Derry, 2002), ya que los alumnos que perciben sensaciones negativas durante la relación con los iguales en ambientes de actividad física, pueden desarrollar ansiedad o sentimientos negativos que les conducirán a ser sujetos no participantes (Kunesh, Hasbrook y Lewthwaite, 1992). En este sentido, las investigaciones coinciden en indicar que el aprendizaje de las experiencias positivas relacionadas con la actividad física durante la adolescencia afecta a los niveles de participación en la edad adulta.

Debemos tener presente que numerosas investigaciones encuentran que uno de los factores contribuyentes a la ausencia de compromiso en el deporte y en el ejercicio hace referencia a malos recuerdos en clase de educación física en toda la educación obligatoria, so- bre todo, los que hacen referencia a una autoimagen carente de competencia atlética, por lo que debemos tratar de incrementar las experiencias y la participación continuada en actividades físicas durante la edad adolescente, lo que contribuirá a incrementar los niveles de participación en actividades físicas durante la edad adulta (Sallis y Patrick, 1994). En la línea reflejada anteriormente en relación a la diferenciación de género, según Brustad (1993) los factores que afectan a la continuidad de participación en actividad físicas de las chicas (sexo que experimenta menor índice de participación respecto al masculino) incluyen el tiempo dedicado al aprendizaje de habilidades, el nivel de disfrute en la participación, la autoestima, los beneficios para la salud recibidos a través de la participación y la percepción de competencia atlética. En definitiva, tal y como lo plantean (Brustad, 1993) las variables correspondientes a diversión, preocupación por sí mismo y competencia física percibida han sido citadas en la bibliografía especializada como importantes determinantes para la continuidad de actividad física en la edad adulta.

\section{En conclusión}

Por medio del deporte se pueden aprender valores que, posteriormente, se pueden desarrollar en todos los ámbitos de la vida, como por ejemplo, la cooperación, la comunicación, el respeto por las reglas y las leyes, la resolución de problemas, liderazgo, honestidad, respeto por los demás (acciones y opiniones), aprender a ganar y a perder, a ser competitivo, mejorar la autoestima, disciplina y confianza en sí mismo (Moncada, 2005). Por consiguiente, el deporte es una actividad social importante que satisface las necesidades lúdicas de los niños y jóvenes junto a su afán competitivo; el cual genera valores como el entusiasmo, lucha apasionada, sentido de convivencia, afanes socializadores, trabajo en equipo y labor comunitaria entre otros (Pila, 1988). Además, creemos que es muy valioso llevar a cabo procesos de socialización y de promoción de valores deportivos en la mayoría de las actividades físicas-deportivas tanto escolares como extraescolares; ahora bien, también debemos reconocer que podrían ser pocos los entrenadores/ directivos (e incluso padres), los que estarían dispuestos a aplicar tiempo en perseguir resultados altruistas en detrimento de contenidos instrumentales y finalistas ganar (Gutiérrez, 1995, 1998), pero creemos que por el bien y la supervivencia del propio deporte se debe volver a la esencia de los valores deportivos. 
Creemos que a través del deporte se deben favorecer los estilos de vida saludables y se debe utilizar como un medio para educar en valores tanto intrapersonales (sacrificio, esfuerzo, frustración, etc.) e interpersonales (solidaridad, cooperación, colaboración, etc.). Sin embargo, debemos tener claro que un enfoque inadecuado, como se está utilizando en la actualidad por parte de los que quieren instrumentalizar el deporte, puede generar la pérdida de valores en los niños ya que se está promoviendo en muchas ocasiones un carácter deshumanizante donde la agresión destructiva hacia el otro competidor es casi una constante (Baratti, 2004).

\section{Referencias bibliográficas}

Abarca, A.; Casterad, J.; Generelo, E. y Clemente, J.A. (2010). Comportamientos sedentarios y patrones de actividad física en adolescentes. Revista Internacional de Medicina y Ciencias de la Actividad Física y el Deporte 10(39), 410-427.

Alonso, J. y Román, J.M. (2005). Prácticas educativas familiares y autoestima. Psicothema 17(1), 76-82.

Amat, M. y Batalla, A. (2000). Deporte y educación en valores. Aula de Innovación Educativa, 91,10-13.

American Academy of Pediatrics (2001). Children, adolescents, and television. Pediatrics 107(2), 423-426.

Arnold, P.J. (1991). Educación física, movimiento y currículum. Madrid: Morata.

Balaguer, I. y García-Merita, M. (1994) Exercici físici benestar psicológico. Anuari de Psicologia, 1, 3-26.

Balibrea, E.; Santos, A. y Lerma, I. (2002). Actividad física, deporte e inserción social: un estudio exploratorio sobre los jóvenes en barrios desfavorecidos. Apunts, educación física y deportes, 69, 106-111.

Bandura, A. (1978). Social learning theory of aggression. Journal of Communication, 28, 12-29.

Baratti, A. (2004). La iniciación deportiva y el deporte escolar. Kinesis, Revista en Ciencias del Deporte, Educación Física y Recreación, 40, 53-55.

Bay-Hinitz, A.; Peterson, R. y Quilitch, H. (1994). Cooperative games: A way to modify aggressive and cooperative behaviors in young children. Journal of Applied Behavior Analysis, 27, 435-446.

144 Berger, B. (1996). Psychological benefits of an active lifestyle: What we know and what we need to know. Quest 48(3), 330-353.

Biddle, S. (1993). Children, exercise and mental health. International Journal of Sport Psychology, 24, 200-216.

Biondi, R.F. (2007). La importancia de la actividad física en el tratamiento de deshabituación a las drogas. Alcmeón 14(2), 82-89.

Bray, S.R. y Kwan, M. (2006). Physical Activity Is Associated with Better Health and Psychological Well-Being during Transition to University Life. Journal of Ameri- can College Health, 55, 77-82.

Bredemeier, B. (1995). Divergence in childrens moral reasoning about issues in daily life and sport specific contexts. International Journal of Sport Psychology, 26, 453-464.

Bredemeier, B.; Weiss, M.; Shields, D. y Shewchuk, R. (1986). Promoting moral grouth in a summer sport camp: The implementation of theoretically grounded instructional strategies. Journal of Moral Education, 15, 212-220.

Brustad, R.J. (1993). Who will go out and play? Parental and psychological influences on children's attraction to physical activity. Pediatric Exercise Science, 5, 210 223.

Buceta, J.M. (1998). Psicología del entrenamiento deportivo. Madrid: Dykinson.

Campos-Izquierdo, A. (2007). Los profesionales de la actividad física y el deporte como elemento de garantía de los servicios. Ciencia, Cultura y Deporte 7(3), 51-57.

Castillo, I. (1995). Socialización de los estilos de vida y de la actividad física: Un estudio piloto con jóvenes valencianos. Tesis de licenciatura inédita. Valencia: Universidad de Valencia.

Cecchini, J.A.; Montero, J. y Peña, J.V. (2003). Repercusiones del Programa de Intervención para desarrollar la responsabilidad personal y social de Hellison sobre los comportamientos de fair-play y el autocontrol. Psicothema 4(15), 631-637.

Cecchini, J.; González, C., Carmona, A. y Contreras, 0. (2004). Relaciones entre clima motivacional, la orientación de meta, la motivación intrínseca, la auto-confianza, la ansiedad y el estado de ánimo en jóvenes deportistas. Psicothema, 16(1); 104-109.

CIS (2010). Hábitos deportivos en España IV. Estudio noo. 2833. Madrid: Centro de Investigaciones.

Consejo de Europa en materia del deporte (2010). El deporte para los niños (Doc. CDDS, 83, 11), vol. II. Madrid: MEC, Consejo Superior de Deportes.

Contreras, 0.; De la Torre, E. y Velázquez, R. (2001). Iniciación deportiva. Madrid: Síntesis.

Cruz, J. (2003). El valor de l.esport en el procés de socialització dels joves», en Escola Catalana, 398,16-18.

Cruz, J. (2004). ¿Es educativo el deporte para jóvenes en edad escolar? En: J. Campos y V. Carratalá (coords.). Las ciencias de la actividad física y el deporte en el marco de la convergencia europea (pp. 69-90). València: Universitat de València.

Cruz, P.J.; Fernández, E. y González, G. (2007). El deporte como método preventivo en el uso de las drogas: teoría y práctica. Wanceulen E.F. Digital, 3, 1-13.

Daley, A. (2008). Exercise and Depression: A Review of Reviews. Journal of Clinical Psychology in Medical Settings 15(2), 140-147.

Daley, A.J. (1997). School based physical activity in the United Kingdom: Can it create physically active adults? Quest, 54, 21-33. 
Danish, S. y Nellen, V.C. (1997). New roles for sport psychologists: Teaching life skills through sport to atrisk youth. Quest, 49, 100-113.

Derry, J.A. (2002). Single-sex and coeducation physical education: perspective of adolescent girls and female physical education teachers (research). Melpómene Journal, 22, 17-28.

Devís, J. (1995). Deporte, educación y sociedad: Hacia un deporte escolar diferente. Revista de Educación, 306, 455-472.

Dirección General de Instituciones Penitenciarias (2005). Estudio sobre salud mental en el medio penitenciario. En: "Programa marco para la atención integral a enfermos mentales en centros penitenciarios". Madrid: Ministerio de Interior.

Elizondo, J.; Guillen, F. y Aguinaga, I. (2005). Prevalencia de actividad física y su relación con variables sociodemográficas y estilos de vida en la población de 18 a 65 años de Pamplona. Rev. Esp. Salud Publica 79(5), 559-567.

Ennis, C.D.; Solmon, M.A.; Satina, B.; Loftus, S.J.; Mensch, J. y McCauley, M.T. (1999). Creating a sense of family in urban schools using the Sport for Peace curriculum. Research Quarterly for Exercise and Sport, 70, 273-285.

Fariña, F.; Vázquez, M.; Mohamed, L. y Novo, M. (2009). Conducta antisocial y psicopatología: estudio de la evolución natural y del riesgo de desviación en una muestra de menores. X Congreso Virtual de Psiquiatría. Interpsiquis. Psiquiatria.com.

Field, T.; Diego, M. y Sanders, C. (2001). Exercise is positively related to adolescents' relationships and academics. Adolescence 36(141), 105-110.

Flores, R. y Zamora, J. (2009). La educación física y el deporte como medios para adquirir y desarrollar valores en el nivel de primaria. Revista Educación 33(1), 133143.

Fox, K.R. (1994). Understanding young people and their decisions about physical activity. British Journal of Physical Education, 25, 15-19.

Fox, K.R. (2000). The effects of exercise on self-perceptions and self-esteem. En: S.J.H. Biddle; K.R. Fox y S.H. Boutcher (eds.). Physical Activity and Psychological Well-Being (pp. 88-118). Londres: Routhledge.

Fraile, A. (1999). Educar con y en el deporte. En: II Jornadas de educadores y educadoras de deporte escolar (comp.) Bizkaia: Diputación floral de Bizkaia. Gráficas Berriz.

Fraile, A. y De Diego, R. (2006). Motivaciones de los escolares europeos para la práctica del deporte escolar. Un estudio realizado en España, Italia, Francia y Portugal. Revista Internacional de Sociología (RIS) 65(44), 85109.

Fundación de Ayuda contra la Droga (FAD) (2009). Club del Buen Deportista. Madrid: FAD.

García Ferrando, M. y Llopis, M. (2011). Ideal democrático y bienestar personal. Encuesta sobre los hábitos deportivos en España 2010. CIS-CSD.
Gibbons, S.L., Ebbeck, V. y Weiss, M.R. (1995). Fair play for kids: Effects on the moral development of children in physical education. Research Quarterly for Exercise and Sport, 66, 247-255.

Giménez, F. y Castillo, E. (2001). La enseñanza del deporte durante la fase de iniciación deportiva. Revista Digital, 6(31). Recuperado de: http://www.efdeportes. com/

Goldberg, D. (1995). Sports and exercise for children with chronic health conditions. Champaign, IL.: Human Kinetics.

Guivernau, M. y Duda, J. (2002). Moral Athmosphere and Athletic Aggressive Tendencies in Young Soccer Players. Journal of Moral Education 31(1), 67-85.

Gutiérrez, M. (1994). Desarrollo y transmisión de valores sociales y personales en educación física y deportes. Premio de Investigación en Ciencias Sociales y Humanidades. Málaga: Unisport.

Gutiérrez, M. (1995). Valores sociales y deporte. La actividad física y el deporte como transmisores de valores sociales y personales. Madrid: Gymnos.

Gutiérrez, M. (1998). Desarrollo de valores en la educación física y el deporte. Apunts: Educación física y deportes, 51, 100-108.

Gutiérrez, M. (2003). Manual sobre valores en la educación física y el deporte. Barcelona. Ed. Paidós

Gutiérrez, M. (2004). El valor del deporte en la educación integral del ser humano. Revista de Educación, 335, 105-126.

Haan, N. (1978). Two moralities in action contexts: Relationship to thought, ego regulation, and development. Journal of Personality and Social Psychology, 36, 286305.

Hastie, P.A. y Buchanan, A.M. (2000). Teaching responsibility through sport education: Prospects of a coalition. Research Quarterly for Exercise and Sport, 71, 25-35.

Heinemann, K. (2002). Esport per a inmigrants: instrument de integració?. Apunts, Educació Física i Esports, 68, 24-35.

Hellison, D. (1995). Teaching responsibility through physical activity. Champaign, Human Kinetics. Recuperado de: http://www.cafyd.com/Revista/art2n3a06.pdf.

Iverson, D.C.; Fielding, J.E.; Crow, R.S. y Christenson, G.M. (1985). The promotion of physical activity in the United States population: The status of programs in Medical, Worksite, Community, and School Settings. Public Health Reports, 2, 212-224.

Jaramillo, L. (2003). La escuela, el señor juego y la formación. Un mundo escolar objetivado por el niño y la niña en torno a la clase de educación física. Una mirada desde la formación. Kinesis, Revista en Ciencias del Deporte, Educación Física y Recreación, 37, 19-25.

Jiménez-Martín, P.J. (2006). Actividad física, deporte y jóvenes en riesgo: reflexiones para la mejora de los programas de intervención. En: E. Gamero et al. (coords.). Violencia, deporte y reinserción social. Estudios sobre ciencias del deporte (pp. 25-44). Madrid: Consejo Su- 
perior de Deportes. Ministerio de Educación y Ciencia.

Kelder, S.H.; Perry, C.L.; Klepp, K. y Lytle, L.L. (1994). Longitudinal tracking of adolescent smoking, physical activity, and food choices behaviour. American Journal of Public Health, 84, 1121-1126.

Knop, P. (1993). El papel de los padres en la práctica deportiva infantil. Málaga, Unisport.

Kohlberg, L. (1969). Stage and sequence: The cognitivedevelopmental approach to socialization. En: D.A. Goslin (ed.). Handbook of socialization theory and research (pp. 347-480). Chicago, Rand McNally.

Kunesh, M.A.; Hasbrook, C.A. y Lewthwaite, R. (1992). Phsyical activity socialization: Peer interactions and affective responses among a sample of sixth grade girls. Sociology of Sport Journal, 9, 385-396.

Locke, L.F. (1996). Dr. Lewin's Little Liver Patties: A Parable About encouraging Healthy Lifestyles. Quest 48(3), 422-431.

Márquez, S.; Rodríguez-Ordax, J. y De Abajo, S. (2006). Sedentarismo y salud: efectos beneficiosos de la actividad física. Apunts. Educación Física Deportiva, 83, 12-24.

Martens, R. (1996). Turning kids on to physical activity lifetime. Quest 48(3), 303-310.

Matsudo, S. (2012). Actividad física: pasaporte para la salud. Rev. Med. Clin. Condes 23(3), 209-217.

Miller, S.; Bredemeier, B. y Shields, D. (1997). Sociomoral education through physical education with at risk children. Quest, 49, 114-129.

Moncada, J. (2005). 2005: Año internacional del deporte y la educación física. Revista Educación 29(2), 235-249.

Moreno, J.A.; Cervelló, E. y Moreno, R. (2008). Importancia de la práctica físico-deportiva y del género en el autoconcepto físico de los 9 a los 23 años. International Journal of Clinical and Health Psychology 8 (1), 171-183.

Moreno, J.A.; Martínez, C. y Alonso, N. (2006). Actitudes hacia la práctica físico-deportiva según el sexo del practicante. Revista Internacional de Ciencias del Deporte, 3(2), 20-43.

Moscoso, D.; Moyano, E.; Biedma, L.; Fernández-Ballesteros, R.; Martín, M.; Ramos, C.; Rodríguez, L. y Serrano, R. (2009). Deporte, salud y calidad de vida. Barcelona: Fundación Obra Social La Caixa.

Negro, C. (1995). Características y peculiaridades del desarrollo de actividades físicas en centros penitenciarios. En: Rodríguez y Moreno (eds.). Perspectivas de actuación en educación Física (pp. 229-246). Universidad de Murcia.

Netz, Y., Meng-Jia, W., Becker, J. y Tenenbaum, G. (2005). Physical Activity and Psychological Well-Being in Advanced Age: A Meta-Analysis of Intervention Studies. Psychology and Aging 20(2), 272-284.

Newcomb, M.B. y Bentler, P.M. (1986). Drug use, educational aspirations, and workforce involvement: The transition from adolescence to young adulthood. American
Journal of Community Psichology, 14, 303-321.

Oja, P. y Telama, R. (1991). Sport for all. Amsterdam, Elsevier Science Publishers.

Pila, A. (1988). Didáctica de la educación física y los deportes. San José, Costa Rica: Editorial Olimpia S.A.

Ramírez, W.; Vinaccia, S. y Ramón, G. (2004). El impacto de la actividad física y el deporte sobre la salud, la cognición, la socialización y el rendimiento académico: una revisión teórica. Revista de Estudios Sociales, 18, 67-75.

Roberts, K. y Brodie, D. (1994). Inner-city sport. Culemborg: Giorano Bruno.

Rossi, R.M.; Becker B. y Lancho, J.L. (2004). La terapia corporal como contexto de desarrollo de la resiliencia: un estudio con adolescentes en situación de riesgo. Lecturas: Educación física y deportes, 105.

Ruiz, F. y García-Montes, M.E. (2002). Retos de la escuela del siglo XXI ante la -sociedad posmoderna del ocio y el tiempo libre. Educar para la mejora de la calidad de vida. Retos. Nuevas perspectivas de educación física, deporte y recreación, 1, 6-8.

Sallis, J.F. y Patrick, K. (1994). Physical activity guidelines for adolescents: A consensus statement. Pediatric Exercise Science, 6, 302-314.

Shields, D. y Bredemeier, B. (1995). Character development and physical activity. Champaign, IL.: Human Kinetics.

Sicilia, A. (2002). La investigación sobre el pensamiento del alumnado. Una revisión desde la educación física. Revista de Educación, 331, 577-613.

Tod, D. y Hodge, K. (2001). Moral reasoning and achievement motivation in sport: A qualitative inquiry. Journal of Sport Behavior 24(3), 307-327.

Tomás, M.; Robles, J. y Giménez, J. (2009). El deporte en las etapas educativas de primaria y secundaria, 14(132). Recuperado de: http://www.efdeportes.com

US Department of Health and Human Services (2000). Healthy People 2010. Washington, DC: Department of Health and Human Services.

Vargas, P. y Orozco, R. (2004). La importancia de la educación física en el currículum escolar. Revista Intercedes, 5(7). Recuperado el 4 diciembre de 2012 de: www.intersedes.ucr.ac.cr

Vázquez, B. (2001). Deporte y educación. En: B. Vázquez et al. (eds.). Bases educativas de la actividad física y el deporte (pp. 333-356). Madrid: Síntesis. 